\title{
Controlling nonlinear longitudinal space charge oscillations for high peak current bunch train generation
}

\author{
P. Musumeci, R. K. Li, and K. G. Roberts \\ Department of Physics and Astronomy, UCLA, Los Angeles, California 90095, USA \\ E. Chiadroni \\ INFN-LNF, Via E. Fermi, 4000044 Frascati, Roma, Italy
}

(Received 19 May 2013; published 8 October 2013)

\begin{abstract}
The evolution of picosecond modulations of the longitudinal profile of an electron beam generated in an rf photoinjector is analyzed and optimized with the goal of obtaining high peak current electron bunch trains at very high frequencies ( $\geq \mathrm{THz}$ ). Taking advantage of nonlinear longitudinal space charge forces, it is found that more than $500 \mathrm{~A}$ peak current $1 \mathrm{THz}$ bunch trains can be generated using a standard 1.6 cell SLAC/UCLA/BNL rf gun. Postacceleration is used to freeze the longitudinal phase space dynamics after one half plasma oscillation. Applications range from tunable narrow bandwidth $\mathrm{THz}$ radiation generation to drivers for high frequency high gradient accelerators.
\end{abstract}

DOI: 10.1103/PhysRevSTAB.16.100701

PACS numbers: 29.27.Bd, 41.75. Ht

\section{INTRODUCTION}

The generation and control of high frequency structures in the longitudinal profile of relativistic electron beams has recently been at the center of attention in particle accelerator research due to its relevance for beam-based high power radiation sources in the $\mathrm{THz}$ spectral range $[1,2]$ and in high gradient high frequency advanced acceleration schemes [3,4].

Different methods to generate ps and sub-ps spaced bunch trains have been proposed and studied. These include intercepting a chirped beam with a properly shaped comb mask in a high dispersion region [5] or using slits before an emittance-exchange beam line to efficiently transfer the transverse modulation to the temporal profile [6]. Another possibility is to illuminate the cathode with a modulated laser pulse then use the velocity bunching technique to rotate the longitudinal phase space and retrieve the density modulation [7]. Difference-frequency echo-based $\mathrm{THz}$ structure generation $[8,9]$ is another very interesting method that just found experimental demonstration even though of slightly more complex implementation since it requires interaction of the beam with two different laser frequencies in an inverse free-electron laser modulator.

All of these methods suffer from increasing the charge in the beam. The modulation blurs and tends to disappear when longitudinal space charge (LSC) forces begin to play a significant role in the beam evolution [10-12]. Considering the beam as a non-neutral relativistic plasma, the time scale for the evolution of the beam distribution

Published by the American Physical Society under the terms of the Creative Commons Attribution 3.0 License. Further distribution of this work must maintain attribution to the author(s) and the published article's title, journal citation, and DOI. under space charge forces is set by the relativistic plasma frequency $\omega_{p}=\sqrt{\frac{e^{2} n_{0}}{\epsilon_{0} m_{0} \gamma^{3}}}$, where $e$ and $m_{0}$ are the electron charge and mass respectively, $n_{0}$ is the electron density, and $\gamma$ the beam relativistic factor. A tighter focusing, a higher current, or a lower beam energy all pose challenges to the preservation of the much-desired microbunch structure.

In a proof-of-principle experiment carried out at the UCLA Pegasus Laboratory a few years ago [13] instead of fighting longitudinal plasma oscillations, we took advantage of the beam self-space-charge forces to demonstrate enhanced bunching and current spiking in the nonlinear LSC regime. It is well known that in linear plasma oscillations an initial perturbation in the longitudinal profile induces an energy modulation which after one half plasma oscillation is converted back to density modulation. In the linear regime (i.e. when the initial density perturbation is small compared to the mean beam density), the dynamics is periodic and the initial condition is retrieved after every plasma period [14,15]. On the other hand, a beam that has a strong initial modulation of its longitudinal charge density (of period $\lambda$ ) will experience a nonlinear plasma oscillation which is characterized by enhanced harmonics of $\lambda$. This leads to the formation of current spikes in the longitudinal charge distribution [16].

Here we want to explore the possibility of using this scheme to generate high frequency beam structures with very high peak currents. Our original experiment was a proof-of-principle demonstration of how the nonlinearity in the longitudinal space charge oscillations could be used to obtain current spiking. As such, it used few pC beam charge and achieved only moderate peak currents $(<10$ Amps). Here we present an optimization of the design of a machine to take advantage of this effect and 
maximize the final peak current. Finally, for many applications it might be useful to accelerate the beam to 10-100 MeV energies before taking advantage of its structure (either for radiation generation or for particle acceleration). With this goal in mind, we study a configuration where a postacceleration structure is introduced in the beam line to freeze the longitudinal phase space dynamics and preserve the THz-bunched structure all the way to high beam energies. Our optimization studies focus on the particular beam line configuration available at the SPARC facility [17], but all of the methods discussed here are fairly general and can be applied to different facilities.

\section{PEAK CURRENT ENHANCEMENT FROM NONLINEAR LONGITUDINAL SPACE CHARGE OSCILLATIONS}

To understand the details of the evolution of a density perturbation in the longitudinal profile of a relativistic electron beam it is convenient to start from a 1D cold fluid model. The equations for the three unknown functions $n(z, t), \Delta \gamma(z, t)$, and $E(z, t)$ describing the electronic density, the deviation from the reference energy, and the LSC field respectively, can be written as

$$
\begin{gathered}
\partial_{t} \Delta \gamma+c \frac{\Delta \gamma}{\gamma^{3}} \partial_{z} \Delta \gamma=-\frac{e E}{m_{0} c}, \\
\partial_{z} E=-\frac{e n}{\epsilon_{0}}, \\
\partial_{t} n+c \partial_{z} n \frac{\Delta \gamma}{\gamma^{3}}=0,
\end{gathered}
$$

where $z$ is the coordinate along the bunch, and we assume $\Delta \gamma \ll \gamma$.

Equations (1)-(3) represent a coupled system of nonlinear partial differential equations. Assuming (for an infinitely long beam) a periodic modulation with period $\lambda=2 \pi / k$, we can expand the solution $A(z, t)=$ $\sum_{m} A_{m}(t) e^{i m k z}$ and, collecting the terms which have equal orders of $m$ in the exponent, we reduce the problem to a system of ordinary differential equations:

$$
\begin{aligned}
\Delta \dot{\gamma}_{m}+\sum_{n} \frac{i n k c}{\gamma^{3}} \Delta \gamma_{m} \Delta \gamma_{m-n} & =-\frac{e E_{m}}{m_{0} c}, \\
\dot{R_{m}}+\sum_{n} \frac{i m k c}{\gamma^{3}} R_{m-n} \Delta \gamma_{m} & =0
\end{aligned}
$$

where the density is normalized to the average beam density and $R_{m}$ are the Fourier series components of $n(z, t) / n_{0}$. Equation (2) reduces to $E_{m}=-\frac{e n_{0} R_{m}}{i \epsilon_{0} m}$. We will be interested in initial conditions where the beam is density modulated at a given spatial frequency $k$, that is $n(z, 0)=n_{0}[1+2 b \cos (k z)]$ or $R_{ \pm 1}=b$. Using this form for the initial conditions, $b=\left|\frac{\int n(z, 0) e^{i k z} d z}{\int n(z, 0) d z}\right|$ corresponds to the usual definition of the bunching factor.

It should be noted that one can introduce a full set of dimensionless coordinates and reduce the problem (4) to a universal set of equations whose evolution is fully determined by the initial conditions:

$$
\begin{aligned}
\partial_{\tau} G_{m} & =-\sum_{n} n G_{n} G_{m-n}+\frac{R_{m}}{m}, \\
\partial_{\tau} R_{m} & =-m \sum_{n} G_{n} R_{m-n},
\end{aligned}
$$

where $\tau=\omega_{p} t$, and $G_{m}=i k c \Delta \gamma_{m} / \omega_{p} \gamma^{3}$.

The formulation of the problem in normalized coordinates allows us to quickly obtain an order of magnitude estimate for the energy spread induced by LSC forces. By solving the linearized system in (5), a small initial perturbation $R_{ \pm 1}(0)=b$ evolves (after one quarter plasma oscillation) into a maximum energy modulation $G_{ \pm 1}(\tau / 4)=R_{ \pm 1}(0)$. In physical units, the maximum (peak-to-peak) energy spread induced by longitudinal space charge forces can then be written as

$$
\Delta \gamma_{\mathrm{LSC}}=4 \frac{\gamma^{3} \omega_{p} b}{c k}
$$

which due to the plasma frequency scaling is proportional to $\gamma^{3 / 2}$, so that a larger energy modulation is obtained for a larger beam energy, higher plasma frequency, and/or longer modulation wavelengths.

It is possible to find an analytical solution for a large initial sinusoidal density modulation using Lagrange coordinates (moving with the fluid) [16]. The solution in the lab frame is obtained by inverting back to Eulerian coordinates. In this general case, the electron density at times $t$ can be written as

$$
n(z, t)=n_{0}\left[1+\cos \left(\omega_{p} t\right) \sum_{m=1}^{\infty} m c_{m}(t) \cos (m k z)\right],
$$

where $\quad c_{m}(t)=\frac{(-1)^{m+1}}{m} \frac{2}{\alpha(t)} J_{m}[m \alpha(t) b] \quad$ and $\quad \alpha(t)=$ $2 \sin ^{2}\left(\omega_{p} t / 2\right)$.

In the nonlinear regime, even if the initial condition is perfectly sinusoidal, the density evolution is characterized by the onset of harmonic components $\left[c_{m}(t)\right]$. The harmonic contributions all sum in phase after one half plasma oscillation causing the beam current to spike up reaching peak values much larger than the initial modulation. For $b=0.25$ the density given by Eq. (7) diverges at $t=\pi \omega_{p}$. This is commonly known as the wave-breaking limit which is the condition where particles with different energies pile up at the same $z$ location. The energy distribution cannot be described anymore by a single-value function of $z$ (i.e. the wave "breaks") and the main approximation of the 1D cold fluid model breaks up. 
In practice as we will see many factors contribute to maintain finite the amplitude of the wave, nevertheless this behavior suggests the application of this mechanism as an efficient way to generate high peak current high frequency bunch trains.

Here we want to obtain an estimate for the actual limits in the current spiking. In Fig. 1 we show the peak current $I_{p}$ (normalized to the average current $I_{\text {avg }}$ ) in the beam as a function of the initial bunching factor $b$ calculated using the solution of the system of equations (4) when a finite number $(N=64)$ of harmonics is included in the sum. The results agree well with the analytical formula from Eq. (7). In the linear regime, i.e., for $b<0.05$, the peak current agrees exactly with the analytical expression $I_{p}=$ $(1+2 b) I_{\mathrm{avg}}$. For $b>0.1$ the behavior of the system becomes more evidently nonlinear.

Using particle tracking simulations it is possible to follow the evolution of the modulation in longitudinal phase space taking into account more details than those included in the simple fluid model. We consider as an example a coasting beam of $5 \mathrm{MeV}(\gamma=10)$ with uniform transverse distribution with $200 \mu \mathrm{m}$ radius and an average current $I_{\text {avg }}=100$ Amps. The corresponding electronic density is just above $10^{13} \mathrm{e}^{-} / \mathrm{cm}^{3}$. The initial density modulation is generated superimposing 20 copies of a Gaussian pulse with a fixed 1 ps spacing $(\lambda=300 \mu \mathrm{m})$. By varying the temporal rms width of these beamlets it is possible to obtain different initial density modulation amplitudes (see Fig. 2). The electron beam is initially assumed very cold $\left(10^{-4}\right.$ slice relative energy spread and $0.1 \mathrm{~mm}$ mrad emittance). For these beam parameters, summarized in Table I, the maximum final peak current for the coasting beam case is obtained for an initial bunching factor slightly larger than 0.25 and it is nearly 13 times larger than the initial average current.

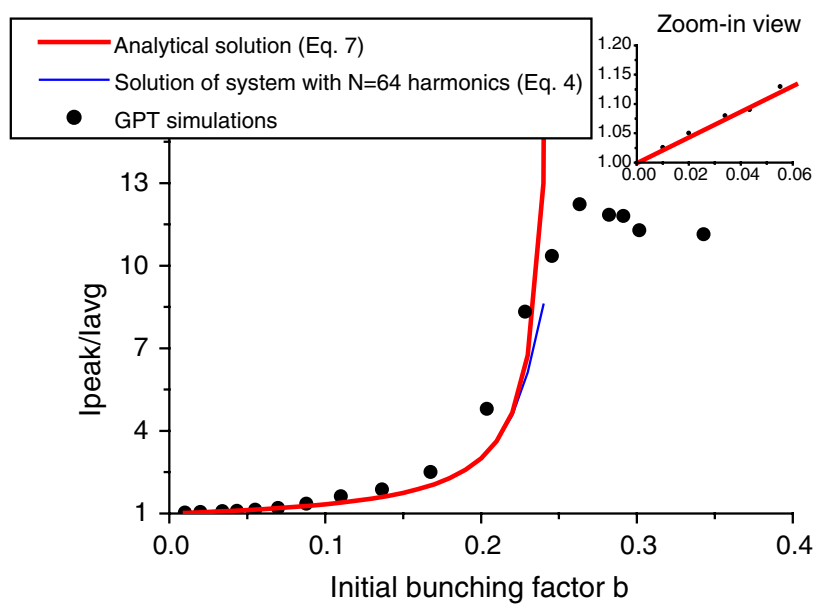

FIG. 1. Nonlinear peak current enhancement as a function of amplitude of initial density modulation. In the inset we show the linear behavior for small initial bunching factor.

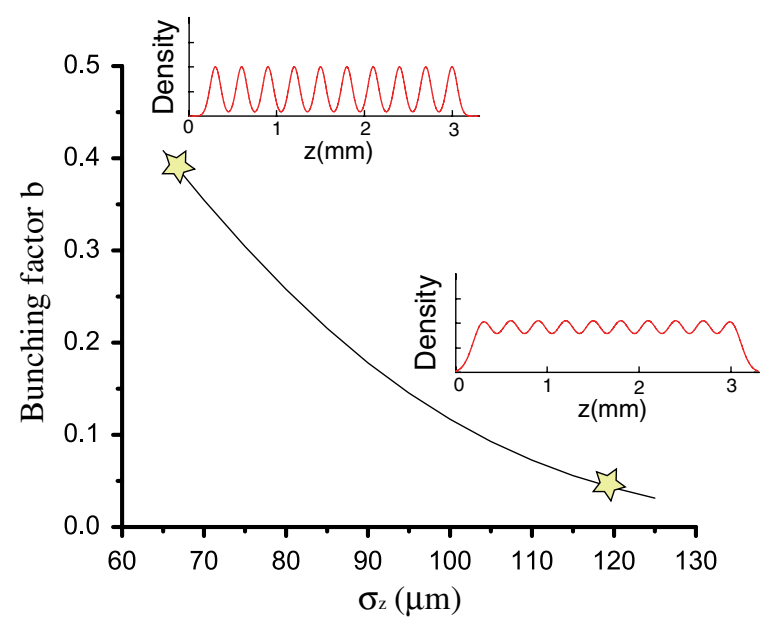

FIG. 2. Bunching factor for a beam distribution generated superimposing 20 Gaussian pulses separated by $300 \mu \mathrm{m}$ as a function of the single pulse rms width.

The results of General Particle Tracer (GPT) simulations [18] for the normalized peak current as a function of the initial bunching factor are reported in Fig. 1. We observe a peak current enhancement (with respect to the average current in the beam) by more than 1 order of magnitude for $b=0.25$ in good agreement with the estimates from the cold fluid model and from the analytical solution. With GPT simulations it is also possible to find out what happens when the wave-breaking condition is reached $(b>0.25)$, exploring the system behavior beyond the cold fluid approximation. For initial bunching larger than the wave-breaking limit, we find no significant increase in the final peak current.

The simulations are performed matching the beam into a focusing channel in Brillouin flow conditions, i.e., with focusing strength $k_{\beta}=k_{p} / \sqrt{2}$. In these conditions three-dimensional effects and strong focusing play a non-negligible role in the longitudinal space charge wave evolution.

The $x-z$ configuration space at the point of maximum current enhancement is shown in Fig. 3. The transverse beam size scallops with the same periodicity of the longitudinal modulation. This is due to the Brillouin equilibrium transport where the transverse beam size depends on the local beam current.

TABLE I. Parameters for coasting beam simulations.

\begin{tabular}{lc}
\hline \hline Energy & $5 \mathrm{MeV}$ \\
Peak current & $100 \mathrm{~A}$ \\
Number of periods & 20 \\
Period & $300 \mu \mathrm{m}$ \\
Total bunch length & $6 \mathrm{~mm}$ \\
Energy spread & $10^{-4}$ \\
Emittance & $0.1 \mathrm{~mm} \mathrm{mrad}$ \\
Transverse radius & $200 \mu \mathrm{m}$ \\
\hline \hline
\end{tabular}




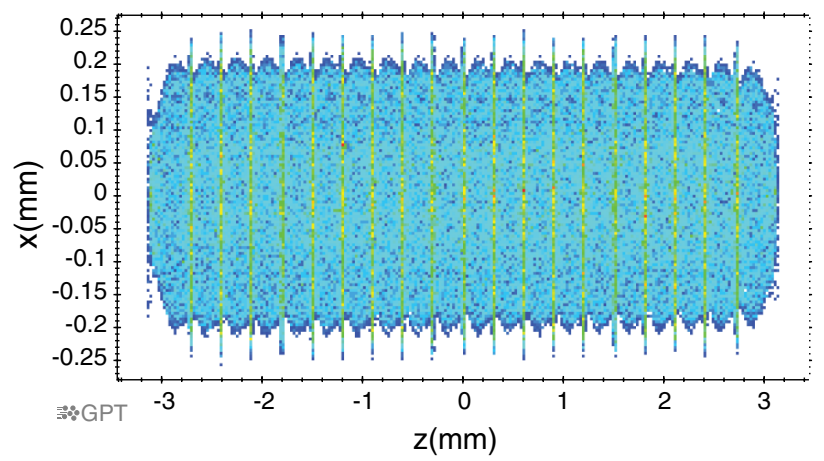

FIG. 3. Configuration space ( $x z$ plane) at maximum current point. The initial bunching factor for this simulation is $b=0.25$.

By solving the Poisson's equation for the periodic longitudinal electric field of a beam with uniform transverse distribution up to radius $R$, we find that the peak field is reduced with respect to the $1 \mathrm{D}$ approximation by a factor $f=1-D K_{1}(D)$ [19], where $K_{1}$ is the first-order modified Bessel function of the second kind and $D=2 \pi R / \lambda \gamma[20]$. This effect lowers the plasma frequency by a factor $\sqrt{f}$. In other words, there are diminishing returns in trying to increase the plasma frequency by tighter beam focusing since, when the transverse spot size becomes much smaller than $\gamma \lambda$, three-dimensional corrections offset the advantage of the higher beam density.

Another effect due to the finite transverse size of the bunch is related to the variation of the longitudinal field across the beam radial profile which tends to smear out the bunching and limit the peak current spike achievable in the nonlinear oscillation. Since the transverse extent of the field is $\gamma \lambda$, a smaller $D$ causes a more homogeneous transverse phase front in the field and hence a larger current enhancement factor. This was confirmed in our simulations. For a relaxed focusing condition and $1 \mathrm{~mm}$ equilibrium transverse radius the maximum peak current enhancement obtained was around 7, almost a factor of 2 smaller than the reference case shown in Fig. 1.

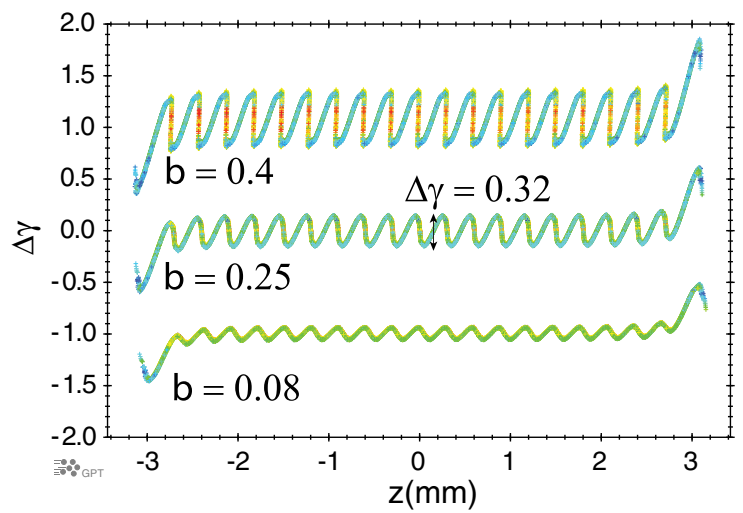

FIG. 4. Longitudinal phase spaces for initial condition below and above the wave-breaking condition.

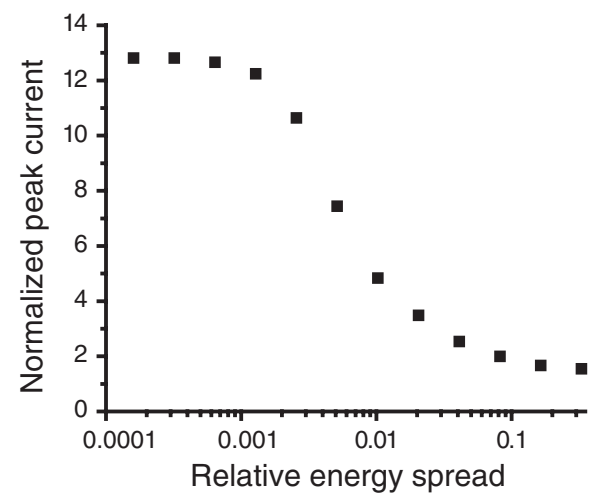

FIG. 5. Peak current vs initial energy spread.

Longitudinal phase spaces for initial density modulations below and above the wave breaking are shown in Fig. 4. The longitudinal space charge field can only be described by our simple model around the central region of the beam where the edge effects are not significant. At the beam head and tail, the periodic boundary conditions on the field and hence the description in terms of plasma oscillations no longer apply.

The simulation results can be used to confirm our initial estimate for the amplitude of the energy modulation obtained using Eq. (6) and allow the study of the finite initial energy spread case. For an initial bunching $b=0.25$, the formula predicts a peak-to-peak energy spread $\Delta \gamma=0.32$ corresponding to a $160 \mathrm{keV}$ energy modulation in excellent agreement with the simulation result.

In Fig. 5 we summarize the behavior of the normalized peak current as we increase the initial uncorrelated energy spread. As expected current spiking decreases for larger initial slice energy spread. We can impose the condition that the longitudinal emittance must be equal or larger than its initial value, to obtain a quick but accurate estimate for the maximum current for any given initial slice energy spread. An upper limit for the current enhancement is the ratio of the space-charge induced energy modulation $\Delta \gamma_{\mathrm{lsc}}$ to the beam uncorrelated energy spread. This is confirmed in the simulations where the current enhancement is observed to drop significantly when the total initial energy spread approaches $\Delta \gamma_{1 \mathrm{lsc}}$.

\section{LONGITUDINAL SPACE CHARGE OSCILLATION FROM THE PHOTOCATHODE TO THE GUN EXIT}

We now proceed to examine the design of an electron injector that maximizes the nonlinear current enhancement from the LSC oscillations. The initial density modulation is obtained by illuminating the cathode in an rf gun with a properly shaped laser pulse. The photocathode driver laser can be modulated using a variety of techniques [21]. We refer the interested reader to the publications on laser pulse train generation since a full discussion of this subject 
would be beyond the scope of this paper. Here we simply assume that we have available a laser pulse with an arbitrary sinusoidal modulation characterized by an amplitude $b$ and wavelength $\lambda$ as previously defined.

When we launch a modulated laser pulse at the cathode, the main difference with the coasting beam case described in the previous section is that the beam undergoes a violent acceleration in the gun and the relativistic factor $\gamma$ is quickly changing along the propagation, instead of being constant as previously assumed. In the initial section where the beam relativistic factor $\gamma$ is still close to unity, the LSC induced energy spread is small in absolute value. This energy modulation cannot be efficiently converted into density modulation after the beam has reached higher energy so that the effect of the acceleration is to strongly damp the plasma oscillation.

To be more quantitative, we can calculate the density modulation at the exit of the gun, since effectively this will be the sole source for subsequent space charge driven oscillations. For small times $t$ we can assume that nonlinear effects will be small and use the linear approximation to calculate the energy spread induced by an initial sinusoidal density modulation of amplitude $b$ :

$$
\Delta \gamma_{1}(t)=\frac{e_{0}^{2} n_{0} b}{m_{0} \epsilon_{0} c k} t
$$

At the end of the gun, the density modulation can be written as the integral of Eq. (8) during the propagation:

$$
\Delta R_{1}=\frac{e_{0}^{2} n_{0} b}{m_{0} \epsilon_{0} c k} \int_{0}^{t_{f}} \frac{t}{\gamma^{3}} d t
$$

Assuming a constant energy increase $\gamma^{\prime}$ in the acceleration region, the bunching at the gun exit where the beam has energy $\gamma_{f}=1+\gamma^{\prime} z$ will be

$$
R_{1}=b\left(1-\frac{e_{0}^{2} n_{0}}{m_{0} \epsilon_{0} c k \gamma^{\prime 2}} \ln \frac{2 \gamma_{f}}{\gamma_{f}+1}\right) .
$$

This behavior can be verified using particle tracking simulation. In GPT simulations we followed the evolution of the beam in the standard SLAC/UCLA/BNL 1.6 cell gun assuming a gradient of $110 \mathrm{MV} / \mathrm{m}$. The initial laser modulation in the simulation had an amplitude of $b=0.28$ and a period of $1 \mathrm{ps}$ for a total pulse length of $16 \mathrm{ps}$. A full list of parameters is reported in Table II. The bunching at the gun

TABLE II. Electron source parameters.

\begin{tabular}{lc}
\hline \hline Gun gradient & $110 \mathrm{MV} / \mathrm{m}$ \\
Launch phase & $25 \mathrm{degrees}$ \\
Charge & $1.6 \mathrm{nC}$ \\
Pulse separation & $1 \mathrm{ps}$ \\
Number of pulses in the train & 16 \\
Average current & $100 \mathrm{Amp}$ \\
Initial density modulation & 0.28 \\
Bunch radius & $1.8 \mathrm{~mm}$ \\
\hline \hline
\end{tabular}
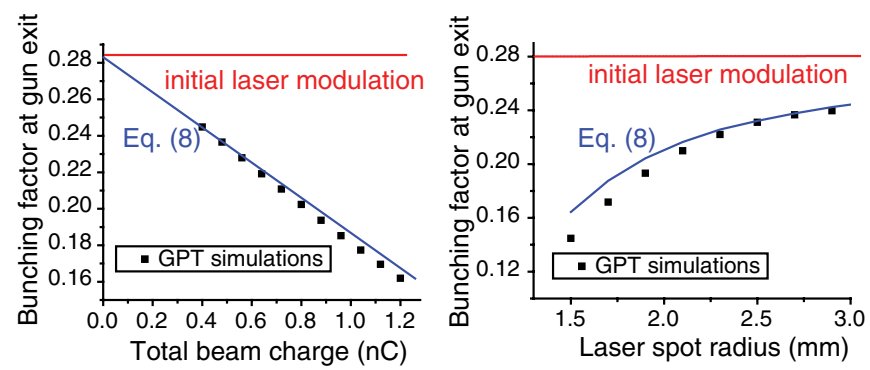

FIG. 6. Bunching factor at the gun exit as a function of laser input parameters and beam charge.

exit as a function of charge and initial laser spot size is shown in Fig. 6 and is found in good agreement with the prediction of Eq. (10).

The optimization of the injection conditions depend on the gradient and final energy, but for currents around 100 Amp and millimeter spot sizes it is possible to obtain a bunching factor at the gun exit only $20 \%$ smaller than the initial one which is set by the laser longitudinal profile modulation. For a larger initial beam density (higher charge or smaller spot size), the plasma phase advance close to the cathode will be too large and the density modulation can never be recovered. We also observe that for lower gun gradients, like the ones employed in DC or high repetition frequency guns, it will be necessary to reduce accordingly (as $\gamma^{\prime 2}$ ) the initial beam density to obtain similarly small plasma phase advances in the region close to the cathode.

In these simulations the injection phase has been set to 25 degrees to minimize the overall correlated energy spread. For different injection phases the bunch length changes and so the plasma phase advance will shift accordingly. The main effect is a compression/stretching of the bunch spacing (and hence of the resonant wavelength) as shown in Fig. 7. The level of tunability of the bunching wavelength for a fixed $1 \mathrm{ps}$ laser pulse modulation period ranges from less than $200 \mu \mathrm{m}$ to more than $300 \mu \mathrm{m}$. The gun phase could serve as a final knob in controlling the final bunching periodicity. It should be noted that the total bunch length is compressed by the same ratio.
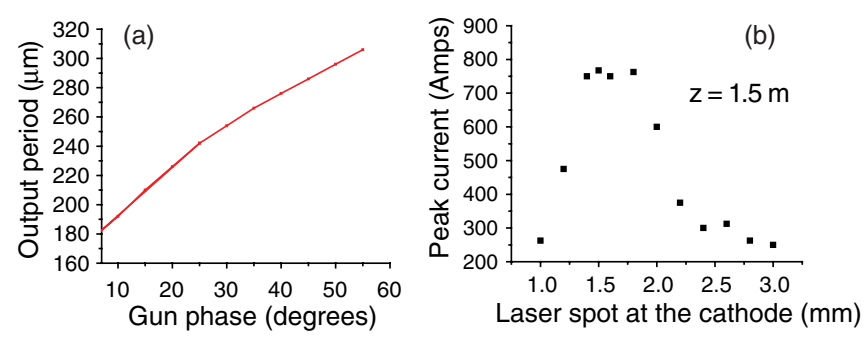

FIG. 7. (a) Peak of bunching factor at the gun exit as a function of laser injection phase showing compression and stretching of the modulation. (b) Peak current at $z=1.5 \mathrm{~m}$ after $1 / 2$ plasma oscillation as a function of input laser spot size. 
After the beam exits the gun, the solenoid is used to focus the beam to a small waist to increase the plasma frequency and accelerate the LSC oscillation. In this case, the $\pi$ plasma phase advance is obtained, rather than by matching the beam into an equilibrium focusing channel, as discussed previously, by a single solenoidal lens with most of the LSC interaction occurring in the region close to the focus. There are at least two reasons behind this choice. First, the higher beam density at the beam waist increases the induced LSC energy spread, highly beneficial in order to obtain the highest possible current enhancement. Second, since the beam is diverging after the focus, the longitudinal phase space evolution slows down and is more easily controlled once the desired density profile is obtained.

Even though the scaling in Eq. (10) drives the design towards a larger initial laser spot, when considering the full space charge oscillation there are many effects that concur to degrade the plasma oscillation process for laser spot sizes larger than few millimeters: (i) The geometric path-length distortion effect is related to the fact that the particles initially born at larger radial offsets are delayed with respect to on-axis particles. It causes curving of the density modulation contours and hence of the space charge field lines. The path-length difference [22] at first order can be expressed as $c \tau_{\text {geometric }}=\left(\alpha+\frac{1}{f c}\right) R^{2}$, where $\alpha$ takes into account the radial dependence of the gun fields (typically on the order of tens of $\mathrm{fs} / \mathrm{mm}^{2}$ ) and $f$ is the focal distance of the solenoidal lens. When $c \tau_{\text {geometric }}$ becomes comparable with the modulation period the current enhancement factor will be strongly reduced. (ii) Another effect is due to the slice energy spread increase. In high frequency guns due to the curvature of the rf fields a larger laser spot is associated with larger slice energy spread. This rf induced energy spread can be estimated as $\delta \gamma_{\mathrm{rf}}=\frac{1}{2}\left(k_{\mathrm{rf}} R\right)^{2}$ and will tend to damp the oscillation and blur the current spikes (see discussion at the end of the previous section). (iii) A larger initial laser spot size also implies larger thermal emittance. The effect of beam emittance is similar to the energy spread and tends to damp the longitudinal space charge oscillations. Marinelli et al. have studied the problem in the linear theory, and extending their results to the nonlinear case [20], it is found that whenever the electron trajectory angles (due to the emittance) are larger than omega ${ }_{p} / k c$ then it becomes very hard to recover the current spike formation after half plasma oscillation.

In our example for $R>2 \mathrm{~mm}$ all these effects become significant and contribute to the loss of coherence of the longitudinal space charge oscillation preventing the spike formation and the current enhancement. We note that the magnitude of these effects is strongly dependent on the modulation period, hence longer wavelengths permit one to reach out to larger beam radius and larger peak currents.

In Table II we report the optimized parameters of an rf gun based electron source to maximize the current enhancement factor at a distance of $1.5 \mathrm{~m}$ from the cathode.

\section{HIGH PEAK POWER TUNABLE NARROWBAND THZ GENERATION}

In this section we discuss a practical implementation of this scheme to the SPARC facility in Frascati, Italy. Details of the facility have been discussed elsewhere [17]. The goal of this study is to propose an experimental set of parameters where the nonlinear longitudinal space charge oscillations are used to generate a high peak current high frequency bunch train. Possible applications of this setup include high peak power $\mathrm{THz}$ generation and driving plasma wakefield acceleration with a bunch train in the nonlinear regime [23].

In practice the scheme works as follows: (i) we start at the cathode with a strong modulation from the temporal profile of the laser, (ii) the plasma phase advance in the gun at low energy is minimized by proper choice of injection parameters, (iii) focusing solenoid is used to squeeze the beam transversely and obtain the required plasma phase advance in the drift region after the gun, (iv) an $S$-band linac is used to lock the longitudinal phase space structure and accelerate to high energies.

In the previous sections we discussed the optimum conditions to preserve significant bunching outside the gun and complete the space charge oscillation with the help of the solenoid in the propagation distance between the gun and the linac. The important element that we add in this section is the accelerating structure located at a distance $1.5 \mathrm{~m}$ from the cathode. The function of this element is to freeze the longitudinal phase space evolution and the plasma oscillation at the point where the maximum peak current is obtained. In our simulations we accelerate the beam at the final energy of $100 \mathrm{MeV}$ where a ps-spaced train of microbunches with more than 500 A peak current is obtained.

The solenoid around the accelerating section is used to control the transverse spot size of the beam and propagate it through the accelerator (see Fig. 8). This causes some (20\%) increase in the transverse emittance which for $\mathrm{THz}$ production applications, usually not very demanding in terms of transverse beam quality, can be tolerated.

The beam longitudinal profile at the exit of the third accelerating section ( $z=12 \mathrm{~m}$ from the cathode) is shown

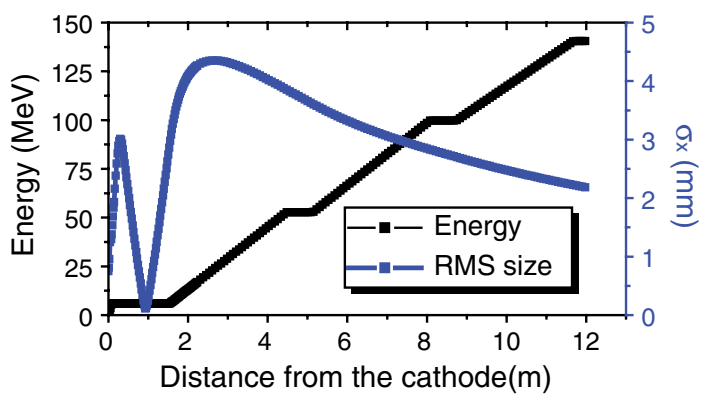

FIG. 8. Energy and transverse spot size of the beam along the SPARC beam line. 
in Fig. 9. The current spiking is not completely uniform along the beam as the head of the beam has a larger slice energy spread. Nevertheless a large number of high current spikes is clearly present. The average beam current is $100 \mathrm{~A}$ and peaks more than $500 \mathrm{~A}$ are obtained. If we take the Fourier transform of the beam distribution we obtain the longitudinal bunch form factor displayed in Fig. 9 which summarizes the ability for the beam to radiate coherent power at a given frequency.

A strong peak at the fundamental wavelength of $270 \mu \mathrm{m}(1.1 \mathrm{THz})$ is obtained. The wavelength is slightly compressed from the initial modulation period at the cathode of $300 \mu \mathrm{m}$. The $2 \mathrm{nd}, 3 \mathrm{rd}$, and 4th harmonics are also evident. It is noticeable that the bunching is still above 0.05 at the 5 th harmonic.

Assuming that the beam is focused to a small spot and that the frequency cut due to the finite dimensions of the target can be neglected, it is possible to estimate the coherent peak $\mathrm{THz}$ energy emitted by this beam as

$$
E_{\mathrm{coh}}=N_{e}^{2} b^{2} \frac{d W_{\mathrm{sp}}}{d \omega} \Delta \omega
$$

where $b$ is the bunching factor, $N_{e}$ is the number of electrons participating to the coherent emission, $\frac{d W_{\mathrm{sp}}}{d \omega}$ is the single particle spectral power which for transition radiation is relatively flat and equal to $\frac{2}{\pi} m_{0} r_{0} c \ln \gamma$, with $r_{0}$ the classical electron radius and $\Delta \omega$ is the linewidth which is equal to 1 over the number of cycles in the pulse train. Using the output of the particle tracking simulations we can estimate a coherent energy of up to $3 \mu \mathrm{J}$ centered around $1.1 \mathrm{THz}$ frequency in $6 \%$ bandwidth.

This must be compared to the energy in the same bandwidth generated when using a single electron pulse fully compressed using rf compression. During the velocity bunching demonstration experiment, a minimum beam size of $210 \mathrm{fs}$ rms was measured for $300 \mathrm{pC}$ beam charge [24]. Assuming a cubic root scaling with charge as typical of photoinjector operation [25], we can estimate the rms bunch length for different charges. For example in single spike free-electron laser simulations Rosenzweig et al. [26] found an optimization that gave $30 \mathrm{fs}$ bunch length at $1 \mathrm{pC}$, roughly in good agreement with the cubic root power scaling. Note that a single beam emits a broadband
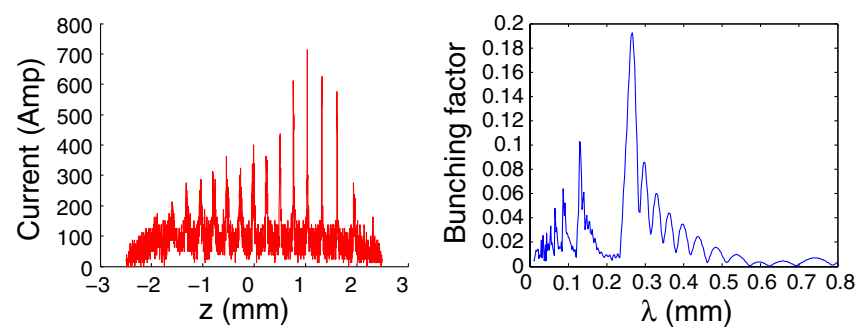

FIG. 9. Density profile of the pulse at $100 \mathrm{MeV}$ and bunching factor. radiation spectrum and narrow band filters must be used to reduce the bandwidth.

In Fig. 10 we plot the radiated energy for the single bunch case and for the bunch train as a function of the beam charge. The single beam bunching factor is obtained assuming a Gaussian shape with an rms bunch length as derived from the cubic root scaling. The bunch train case assumes that the same bunching factor shown in Fig. 9 could be obtained for different beam charges using the schemes proposed in this paper. We performed a tolerance analysis for the nonlinear space charge oscillation case by letting the beam charge vary by $\pm 3 \%$ and the linac phase randomly fluctuate by 3 degrees. The amount of radiation generated will be reduced, but the bunching factor only changes by less than $10 \%$. We indicate with the shaded areas in the figure the possible variation in $\mathrm{THz}$ power due to these machine jitters.

It stems from this analysis that the harmonic content arising from the nonlinear space charge dynamics is especially important since the velocity bunching technique has severe limitations when trying to generate sub-50 fs bunches with large amount of charge. Above few $\mathrm{THz}$ the methods proposed in this paper are the most viable solution to generating large amount of coherent power.

Other radiation mechanisms to generate $\mathrm{THz}$ radiation, such as undulator radiation or Cherenkov radiation in dielectric loaded or corrugated structures, can be used to obtain narrow bandwidth $\mathrm{THz}$ radiation. The analysis to calculate the amount of radiation generated proceeds in the same way replacing the single particle transition radiation spectrum $s \frac{d W_{\mathrm{sp}}}{d \omega}$ with the one characteristic of the mechanism under consideration. The advantage of our proposed method is in the larger coherent form factor which multiplies the single particle spectrum. Taking advantage of nonlinear longitudinal space charge oscillations enables

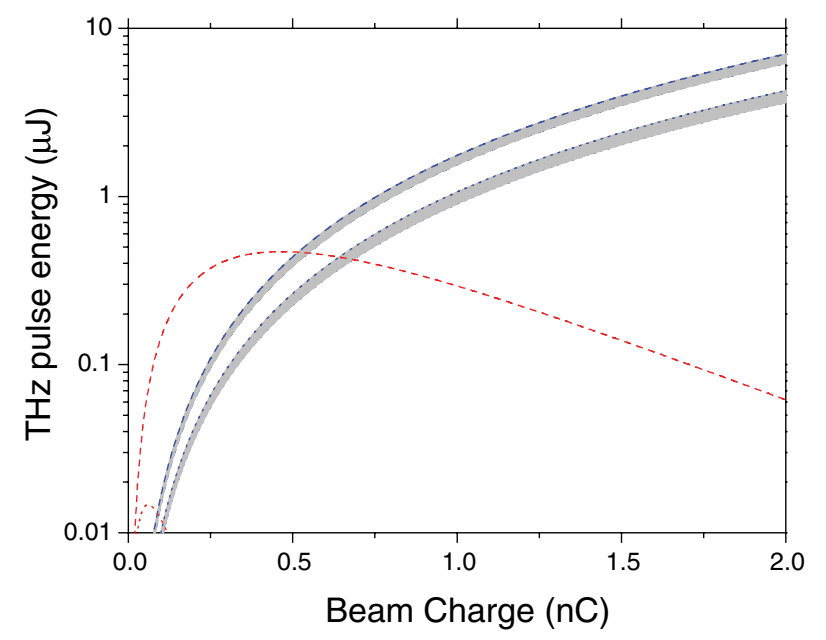

FIG. 10. Energy generated in a 1/16 bandwidth around a central frequency at $1.1 \mathrm{THz}$ (dashed) and $2.2 \mathrm{THz}$ (dotted) comparing the single bunch after bandwidth selecting filters (red) and the pulse train method (blue). 
(especially at higher frequency) obtaining a larger bunching factor than what can be achieved using different preparation techniques for the electron beam.

We also note that the 500 A peak current achieved in simulations is 5-7 times larger than what used at BNL for plasma or wakefield drivers. In this paper we have focused on $\mathrm{THz}$ generation, but the beams generated here can drive large amplitude wakefields.

\section{CONCLUSIONS}

This paper discusses the possibility of taking advantage of nonlinear longitudinal space charge oscillations to create high peak current high frequency bunch trains. It is shown that the limitations to the maximum peak current enhancement originate from the intrinsic energy spread and from 3D effects. We discuss a practical design for a source based on this mechanism analyzing in detail the initial propagation region close to the cathode. The optimization of the design drives the parameter choice towards a high peak extraction field and relatively low beam density to minimize the plasma phase advance at low energies which is found not effective in creating density modulation. We analyze the possibility of using a linac accelerating structure to freeze the nonlinear space charge oscillation and preserve the spiky peak current structure to high energies where it can be used for $\mathrm{THz}$ power production or to drive high frequency accelerating structures.

The combination of the nonlinear analysis with longitudinal space charge amplifier concepts can be extended to higher frequencies, and the analysis shown in this paper points towards the challenges in doing so. In particular slice energy spread and three-dimensional effects will make the control of space charge oscillation above few $\mathrm{THz}$ particularly challenging. The harmonic content characteristic of the nonlinear oscillations could be used to increase the spectral reach of the bunch form factor. In principle, a second magnetic compression stage at higher energy could also be used to further compress the bunch and increase/tune the frequency of the periodicity and hence the peak of the coherent emission.

\section{ACKNOWLEDGMENTS}

This work was partially supported by DOE Grants No. DE-FG02-92ER40693 and No. DE-FG0207ER46272.

[1] Y. Shen, X. Yang, G. L. Carr, Y. Hidaka, J. B. Murphy, and X. J. Wang, Phys. Rev. Lett. 107, 204801 (2011).

[2] A. Gover, Phys. Rev. ST Accel. Beams 8, 030701 (2005).
[3] C. Jing, A. Kanareykin, J. Power, M. Conde, Z. Yusof, P. Schoessow, and W. Gai, Phys. Rev. Lett. 98, 144801 (2007).

[4] G. Andonian, O. Williams et al., Appl. Phys. Lett. 98, 202901 (2011).

[5] P. Muggli, V. Yakimenko, M. Babzien, E. Kallos, and K. P. Kusche, Phys. Rev. Lett. 101, 054801 (2008).

[6] Y.E. Sun, P. Piot, A. Johnson, A. H. Lumpkin, T. J. Maxwell, J. Ruan, and R. Thurman-Keup, Phys. Rev. Lett. 105, 234801 (2010).

[7] M. Boscolo, M. Ferrario, I. Boscolo, F. Castelli, and S. Cialdi, Nucl. Instrum. Methods Phys. Res., Sect. A 577, 409 (2007).

[8] D. Xiang and G. Stupakov, Phys. Rev. ST Accel. Beams 12, 080701 (2009).

[9] M. Dunning, C. Hast, E. Hemsing et al., Phys. Rev. Lett. 109, 074801 (2012).

[10] Y. Li and K. J. Kim, Appl. Phys. Lett. 92, 014101 (2008).

[11] J. G. Neumann, R. B. Fiorito, P. G. O'Shea, H. Loos, B. Sheehy, Y. Shen, and Z. Wu, J. Appl. Phys. 105, 053304 (2009).

[12] J. R. Harris, J. G. Neumann, K. Tian, and P. G. O'Shea, Phys. Rev. E 76, 026402 (2007).

[13] P. Musumeci, R. K. Li, and A. Marinelli, Phys. Rev. Lett. 106, 184801 (2011).

[14] E. A. Schneidmiller and M. V. Yurkov, Phys. Rev. ST Accel. Beams 13, 110701 (2010).

[15] A. Gover, A. Nause, E. Dyunin, and M. Fedurin, Nat. Phys. 8, 877 (2012).

[16] R. C. Davidson, in Methods in Nonlinear Plasma Theory (Academic Press, New York, 1972).

[17] M. Ferrario et al., Nucl. Instrum. Methods Phys. Res., Sect. B 309, 183 (2013).

[18] http://www.pulsar.nl/gpt.

[19] M. Venturini, Phys. Rev. ST Accel. Beams 11, 034401 (2008).

[20] A. Marinelli, E. Hemsing, and J. B. Rosenzweig, Phys. Plasmas 18, 103105 (2011).

[21] M. Boscolo, M. Ferrario, C. Vaccarezza, I. Boscolo, F. Castelli, and S. Cialdi, Int. J. Mod. Phys. B 21, 415 (2007); S. Zhou, D. G. Ouzounov, H. Li, I. V. Bazarov, B. M. Dunham, C. K. Sinclair, and F. W. Wise, Appl. Opt. 46, 8488 (2007); A. S. Weling, B. B. Hu, N. M. Froberg, and D. H. Auston, Appl. Phys. Lett. 64, 137 (1994).

[22] M. J. de Loos, S. B. van der Geer, Y. M. Saveliev, V. M. Pavlov, A. J. W. Reitsma, S. M. Wiggins, J. Rodier, T. Garvey, and D. A. Jaroszynski, Phys. Rev. ST Accel. Beams 9, 084201 (2006).

[23] E. Chiadroni et al., Rev. Sci. Instrum. 84, 022703 (2013).

[24] M. Ferrario et al., Phys. Rev. Lett. 104, 054801 (2010).

[25] J.B. Rosenzweig and E. Colby, in Proceedings of the Particle Accelerator Conference, Dallas, TX, 1995 (IEEE, New York, 1995), p. 957.

[26] J. B. Rosenzweig, Nucl. Instrum. Methods Phys. Res. 593, 39 (2008). 